\title{
A ternary hybrid system based on combination of mesoporous silica, heteropolyacid and double-layered clay: an efficient catalyst for the synthesis of 2,4-dihydro- $3 H$-pyrazol-3-ones and pyranopyrazoles in aqueous medium: studying the effect of the synthetic procedure on the catalytic activity
}

\author{
Samahe Sadjadi ${ }^{1}$, Majid M. Heravi ${ }^{1}$, Vahideh Zadsirjan ${ }^{1} \&$ Vahid Farzaneh ${ }^{2}$ \\ 1- Gas Conversion Department, Faculty of Petrochemicals, Iran Polymer and Petrochemical Institute, \\ PO Box 14975-112, Tehran, Iran \\ 2- Department of Chemistry, School of Science, Alzahra University, PO Box 1993891176, Vanak, \\ Tehran, Iran
}

\begin{abstract}
SBA/hydrotalcite/heteropolyacid nanocomposite is synthesized via a novel procedure in which the as-prepared heteropolyacid-loaded SBA-15 was impregnated with calcined hydrotalcite. The ternary hybrid system was characterized by using SEM/EDS, XRD, BET, TPD, TGA, FTIR and ICP-AES and also used as an efficient catalyst for the synthesis of 2,4-dihydro-3Hpyrazol-3-one derivatives via the reaction of arylaldehydes and 5-methyl-1Hpyrazol-3(2H)-one under reflux condition. Moreover, the catalytic activity of this catalyst was confirmed for the one-pot four-component reaction of aryl aldehydes, ethylacetoacetate, malononitrile and hydrazine hydrate/phenyl hydrazine in aqueous media for the synthesis of pyranopyroles. The investigation of the effect of the synthetic procedure and calcination of the
\end{abstract}


Source: Sadjadi, S., Heravi, M.M., Zadsirjan, V. et al. A ternary hybrid system based on combination of mesoporous silica, heteropolyacid and double-layered clay: an efficient catalyst for the synthesis of 2,4-dihydro-3H-pyrazol-3-ones and pyranopyrazoles in aqueous medium: studying the effect of the synthetic procedure on the catalytic activity. Res Chem Intermed 44, 6765-6785 (2018). https://doi.org/10.1007/s11164-018-3521-y

hydrotalcite on the catalytic activity of the catalyst established that this factor does not exert a marked effect on the catalytic activity. The present procedures benefit from diverse advantages, including high yields, simplicity, mild reaction conditions and short reaction times. Moreover, this catalyst was reusable for up to five reaction runs and the HPA leaching was suppressed.

Keywords: 2,4-Dihydro-3H-pyrazol-3-ones, Pyranopyrazoles, Catalyst, Hybrid, Heteropolyacid, SBA, Hydrotalcite 